\title{
The therapeutic outcome of intravenous steroid therapy for active Graves' orbitopathy is influenced by the time of response but not polymorphisms of the glucocorticoid receptor
}

\author{
Guia Vannucchi ${ }^{1}$, Danila Covelli ${ }^{1}$, Irene Campi ${ }^{1}$, Daniele Origo ${ }^{1}$, Nicola Currò ${ }^{2}$, \\ Valentina Cirello' ${ }^{1}$, Davide Dazzi ${ }^{3}$, Paolo Beck-Peccoz ${ }^{1}$ and Mario Salvi ${ }^{1}$ \\ 'Endocrine Unit, Department of Clinical Sciences and Community Health, Graves' Orbitopathy Center, \\ Endocrinology and ${ }^{2}$ Department of Ophthalmology, University of Milan and Fondazione Cà Granda IRCCS, \\ Via Sforza, 35, 20122 Milan, Italy and ${ }^{3}$ Division of Internal Medicine, Ospedale di Fidenza, Fidenza, Italy
}

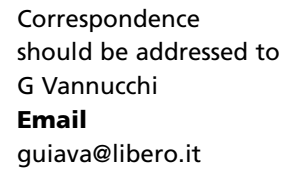

\section{Abstract}

Background: Glucocorticoids are the mainstay of immunosuppression for active moderate-severe Graves' orbitopathy (GO). Aim: To analyze the response to therapy and the contribution of glucocorticoid receptor (GR) gene polymorphisms to the therapeutic outcome of intravenous glucocorticoids (IVGC) in active moderate-severe GO.

Methods: We have studied 58 patients treated with $7.5 \mathrm{~g}$ i.v. methylprednisolone (cumulative dose). Ophthalmological assessment was performed at baseline and at 6-8, 12-16, and 24-30 weeks after the first infusion. Three GR gene polymorphisms, ER22/23EK, N363S, and BCL1, which have been associated to variable sensitivity to steroids, were studied in $43 / 58$ patients. The therapeutic outcomes defined as: i) reduction of the clinical activity score (CAS) $\geq 2$ points or ii) reduction of proptosis $\geq 2 \mathrm{~mm}$ or iii) improvement of diplopia according to the Gorman score were also studied in relation to treatment schedule, age, gender, duration of thyroid or GO, smoking habits, and serum TSH-receptor autoantibodies levels.

Results: In total, $70 \%$ of patients responded and had GO inactivation (CAS <4) as early as 6-8 weeks. At 12-16 weeks, the proportion of patients who became inactive increased by another $10 \%$ up to a total of $80 \%$. ER22/23EK and N363S polymorphisms were present only in about $7 \%$, while the Bcl1 variant was present in $30 \%$ of patients; no significant association of any of the GR polymorphisms with either the therapeutic response or the occurrence of side effects was observed.

Conclusions: Most patients with active GO respond to IVGC as early as 6-8 weeks of therapy and the analyzed GR polymorphisms do not influence the therapeutic effect of steroids. Questions arise about the need of continuing therapy up to 12 weeks in nonresponders. We suggest that these patients may be switched to other treatments alone or in combination with steroids.

\section{Introduction}

Glucocorticoids represent the mainstay of immunosuppression for active moderate-severe Graves' orbitopathy (GO). Treatment effectiveness is limited by great inter-individual variability that may potentially lead to treatment failure or drug-induced toxicity. A satisfactory response to therapy has been reported in as many as
$75-80 \%$ of patients (1) when glucocorticoids are administered intravenously (intravenous glucocorticoid, IVGC) compared with $60 \%$ of patients with oral administration $(2,3)$. The observations of serious cardiovascular and hepatic morbidity after IVGC have suggested a reduction in the pulse doses of methylprednisolone; to date,
(C) 2014 European Society of Endocrinology Printed in Great Britain
Published by Bioscientifica Ltd. 
a cumulative dose of $8 \mathrm{~g}$ is considered rather safe, as long as strict monitoring of liver function tests, hepatitis virus markers, serum glycemia, and blood pressure is carried out. A very recent multicenter clinical trial of EUGOGO (4) has shown that the optimal treatment schedule and dose of IVGC is $830 \mathrm{mg}$ methylprednisolone administered weekly for 6 weeks followed by $415 \mathrm{mg}$ for another 6 weeks, for a cumulative dose of $7.47 \mathrm{~g}$, resulting in a short-term and transient advantage over lower doses but associated with a slightly greater toxicity. Based on these findings, the authors have suggested that this dose regimen may be used in more severe cases of GO, while an intermediate dose regimen may be used in most cases with moderate disease.

Up to now, there are no baseline parameters useful to predict either the patient's response to steroid therapy or the risk of side effects. It has been reported that an early response to IVGC may predict successful outcome, and treatment response is inversely related to disease duration (5). Moreover, while there is consensus on the dose-dependent effect of IVGC on liver enzymes elevations during therapy, the role of hepatic steatosis and diabetes as risk factors for liver damage during pulse IVGC treatment is still controversial $(6,7,8)$. Furthermore, in a recent report, the detection of serum viral hepatitis markers before and after IVGC therapy did not influence the reactivation of hepatitis B even after relatively high therapeutic doses (9). Several genetic studies suggest that both germline and somatic mutations of the glucocorticoid receptor ( $G R$ (NR3C1)) gene may affect steroid sensitivity and its role in several human diseases. Nucleotide sequence variants of GR are reported to modify the intensity of GC sensitivity, both increasing (N363S/BCl1) or decreasing (ER22/23EK) sensitivity to glucocorticoids $(10,11,12)$. In addition, a recent study has demonstrated that the N363S polymorphism is significantly associated with a more frequent hepatotoxicity in pediatric patients affected by lymphoblastic leukemia (13). In this work, we have studied in a considerably large series of patients with moderate-severe GO treated with IVGC, the time of the therapeutic response, and how the presence of polymorphisms of the GR gene may influence it.

\section{Subjects and methods}

\section{Patients}

We studied 58 patients ( 44 females and 14 males, mean \pm S.E.M. age $52.8 \pm 12.4$ years) with active moderate-severe $\mathrm{GO}$, treated with IVGC pulse therapy in our institution from 2003 to 2010. Fifty-three patients had Graves' disease (GD), while three patients had Hashimoto's thyroiditis. Twenty-nine patients (50\%) were smokers. Mean \pm s.E.M. GD and GO duration, determined as the time elapsed from initial diagnosis to the first visit, was $18.9 \pm 21.6$ and $10.9 \pm 12.6$ months respectively. At the time of steroid treatment, 47 patients were euthyroid, 26 on anti-thyroid therapy, 13 on $\mathrm{L}-\mathrm{T}_{4}$, and eight patients without therapy; seven patients were mildly hyperthyroid and four patients mildly hypothyroid. Five patients had received previous radioiodine treatment, three more than 12 months before and two 1 and 2 months before, respectively, in another center. Baseline characteristics of patients are shown in Table 1. Patients underwent endocrinological evaluation, biochemical analysis, and ophthalmological examination according to a recent EUGOGO consensus statement (14). The ophthalmological assessment included lid fissure and Hertel measurements, color vision, cover test, HessLancaster screen, visual acuity, tonometry, fundus examination, and visual field. Soft tissue involvement was graded according to the Colour Atlas available at www.eugogo.eu (15); activity of GO was classified by the clinical activity score (CAS) (16): GO was considered active

Table 1 Baseline clinical and biochemical characteristics of patients with active moderate-severe Graves' orbitopathy (GO).

\begin{tabular}{|c|}
\hline $\begin{array}{l}\text { Number of patients } \\
\text { Gender (male/female) } \\
\text { Age (years) } \\
\text { No. of smokers (\%) } \\
\text { Previous steroid therapy (n) } \\
\text { Clinical activity score } \\
\text { GD durationb } \\
\text { GD duration }{ }^{\text {d }} \\
\text { GO duration }{ }^{\text {b }} \\
\text { GO duration }{ }^{\text {d }} \\
\text { Proptosis OD (mm) } \\
\text { Proptosis OS (mm) } \\
\text { Fissure OD (mm) } \\
\text { Fissure OS (mm) } \\
\text { Gorman score (absent, intermittent, } \\
\quad \text { inconstant, constant) (n) } \\
\text { TSH (mU/l) (n.v. 0.28-4.3) } \\
\text { FT }_{4} \text { (pg/ml) (n.v. 8-17) } \\
\text { FT } \text { (pg/ml) (n.v. 2-5) } \\
\text { TRAb (U/l) (n.v. <1.5) } \\
\text { Glycemia (mg/dl) (n.v. 60-100) } \\
\text { AST (mg/dl) (n.v. 5-32) } \\
\text { ALT (mg/dl) (n.v. 5-31) } \\
\text { GGT (mg/dl) (n.v. 5-36) }\end{array}$ \\
\hline
\end{tabular}

58

$14 / 44$

$52.8+12.4^{\mathrm{a}}$

$29(50)$

13

$4.4+0.1^{\mathrm{a}}$

$38(4-97)^{\mathrm{c}}$

$18(2-117)^{\mathrm{c}}$

$12(4-81)^{c}$

$11(1-75)^{\mathrm{C}}$

$23.0 \pm 0.4^{\mathrm{a}}$

$22.7+0.4^{\mathrm{a}}$

$12.0+0.3^{\mathrm{a}}$

$12.0 \pm 0.3^{\mathrm{a}}$

$16 / 11 / 14 / 17$

$2.76+0.4^{a}$

$14.8 \pm 1.0^{\mathrm{a}}$

$7 \pm 0.7^{\mathrm{a}}$

$19.1 \pm 3.9^{a}$

$91.4+3.6^{\mathrm{a}}$

$19.4+0.9^{a}$

$20.8 \pm 1.4^{\mathrm{a}}$

$22.7 \pm 2.3^{\mathrm{a}}$ n.v., normal value.

${ }^{a}$ Values are shown as mean + s.E.M.

${ }^{b}$ Time elapsed from symptoms appearance to the first visit.

'Values are shown as median and range.

dime elapsed from initial diagnosis to the first visit. 
if at baseline the patient had CAS $>3 / 7$ (seven patients) or $4 / 10$ (50 patients). One patient with $\mathrm{CAS}=2$ was included because of predominant motility involvement. Among severity parameters, proptosis, lid fissure, and Gorman motility score were assessed. Out of 58 patients, sixteen who did not have diplopia at baseline were classified as having moderate to severe $\mathrm{GO}$ because of marked proptosis (Hertel 23-29 mm in 10/16 patients) and marked soft tissue involvement associated with impairment of ductions assessed with Goldman perimeter. The study was approved by the ethics committee of our Hospital and informed consent was obtained from all the patients.

\section{Serology}

Serum $\mathrm{FT}_{4}, \mathrm{FT}_{3}$, and $\mathrm{TSH}$ concentrations were measured using the electrochemiluminescent immunoassay (ECLIA, Roche Diagnostics), while serum TSH-receptor autoantibodies (TRAb) were measured as TSH-binding inhibitory immunoglobulins using a second-generation TRAK human lumitest (Thermo Fisher, AG, Henningsdorf, Berlin, Germany). Serum glucose, aminotransferases and gammaglutamyltransferase were measured at each point of follow-up and expressed as $\mathrm{mg} / \mathrm{dl}$. Serology for hepatitis B virus (HBV) and hepatitis $\mathrm{C}$ virus (HCV) was monitored throughout the treatment course.

\section{Genetic analysis}

Genetic analysis of the $G R$ gene was carried out in 43 patients in order to study the presence of three known GR polymorphisms, N363S, E22R/E23K, and Bcl1. After amplification by PCR with specific primers of exon 2, enzymatic digestion with the restriction enzyme Tsp509I and direct sequencing were performed for the N363S and the E22R/E23K polymorphism respectively. The Bcl1 polymorphism was studied by allele-specific amplification with two couples of primers, each of them specific for the polymorphic sequence.

\section{IVGC therapy}

I.v. methylprednisolone was administered weekly for 16 consecutive weeks until 2010 in 42 patients $(500 \mathrm{mg} /$ day weekly for 14 weeks followed by $250 \mathrm{mg}$ weekly for 2 weeks) and for 12 weeks from 2010 in 14 patients (830 mg/day weekly for 6 weeks followed by $415 \mathrm{mg} /$ day weekly for 6 weeks), with both treatment schedules administering a total cumulative dose of $7.5 \mathrm{~g}$. Two patients underwent orbital surgical decompression because of deterioration of vision that led to a diagnosis of optic neuropathy. One patient after the first 8 weekly infusions of $500 \mathrm{mg}$ of i.v. methylprednisolone switched to i.v. dexamethasone $80 \mathrm{mg}$ for eight infusions, $40 \mathrm{mg}$ for 2 weeks, because she became allergic to methylprednisolone. Patients were studied before therapy, at 6-8 weeks, at the end of methylprednisolone infusions (12-16 weeks), and at 24-30 weeks of follow-up. While receiving steroids, all patients were also treated with proton-pump inhibitor in order to prevent gastric complications and with supplementation of calcium, vitamin D, and bisphosphonates for the prophylaxis of steroid-induced osteoporosis.

\section{Therapeutic outcomes}

GO activity (primary end point) - Effective response to therapy was defined as the decrease in the CAS of at least two points and GO inactivation as the decrease in the CAS to $<4 / 10$ points.

GO severity $\downarrow$ The decrease in proptosis and lid fissure after therapy had to be of at least $2 \mathrm{~mm}$ to be significant. Eye motility improvement was defined as a change of at least one class of the Gorman score (from constant to inconstant, inconstant to intermittent, or intermittent to normal).

Safety $\downarrow$ Side effects were monitored and classified as major (uncontrolled diabetes, occurrence of major depression, severe infections, and any adverse effect leading to IVGC discontinuation) and minor (worsening of liver function, insomnia, dyspepsia, flushing, myalgia, asthenia, nausea, development or worsening of diastolic hypertension).

\section{Statistical analysis}

All values are expressed as mean \pm s.E.M. Analysis by $\chi^{2}$ test or Mann-Whitney $U$ test was applied as appropriate and performed using SPSS 8.0 for Windows. Statistical significance was defined as $P<0.05$. The changes in CAS and proptosis values in response to IVGC therapy were analyzed by repeated measures ANOVA. The nonparametric variables were analyzed by Kruskal-Wallis test.

\section{Results}

\section{Time course of the therapeutic effect of IVGC}

The CAS decreased significantly at each assessment time point during IVGC pulse therapy $(P<0.001)$. In detail, baseline CAS was $4.4 \pm 0.9$ and significantly decreased 


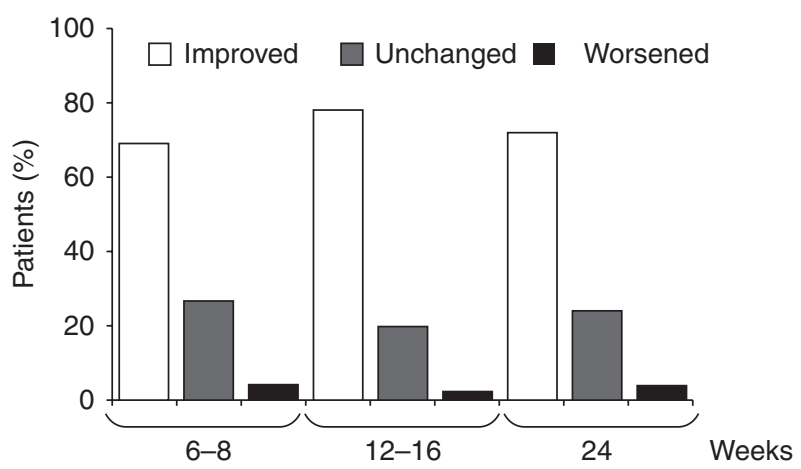

Figure 1

Proportion of patients with active Graves' orbitopathy responding to IVGC at 6-8 weeks, at the end of therapy (12-16 weeks), and at 24 weeks of follow-up.

to $2.2 \pm 1.5(P<0.03)$ at $6-8$ weeks, $1.8 \pm 1.5(P<0.001)$ at $12-16$ weeks, and $1.9 \pm 1.7(P<0.03)$ at 24 weeks of followup. At the end of GC therapy (12-16 weeks), the percentage of patients whose GO inactivated was $80 \%$ $(47 / 58)$ and $70 \%(40 / 58)$ at 24 weeks of follow-up because GO had reactivated in $11 / 58$ patients (20\%) after IVGC therapy withdrawal (Fig. 1). At 6-8 weeks, $70 \%$ of patients responded to therapy with a significant decrease of the CAS. All but eight patients responding to treatment showed GO inactivation (reduction of CAS below 4/10). In the eight patients (17\%) who did not respond to IVGC, the disease remained active (Fig. 2A). By the end of infusions, five of these patients showed a late response to therapy and disease inactivation, three patients had no improvement but worsening of soft tissue involvement during follow-up and two of these subsequently developed optic neuropathy (2/8) requiring surgical orbital decompression (Fig. 2B). As two different infusion protocols had been used in this study, we have compared the mean methylprednisolone dose administered at 6-8 weeks in patients who became inactive (4.3 g methylprednisolone (MP)) with that of patients with persistently active disease ( $4.1 \mathrm{~g} \mathrm{MP})$, and we found no significant difference $(P=\mathrm{NS})$. At the end of IVGC therapy (12-16 weeks) only an additional $10 \%(5 / 43)$ of patients had GO inactivation (Fig. 2A).

\section{Analysis of factors influencing the therapeutic response}

Genetic analysis of the $G R$ gene in $43 / 58$ patients (33 responders and ten nonresponders) has found the N363S and the E22R/E23K heterozygous polymorphisms in two and three patients, respectively, and the heterozygous Bcl1 variant in 13 patients. Two patients showed Bcl1 homozygous polymorphism. A GR polymorphism was found in 19/33 responders (57.5\%) (E22R/E23K: $n=3$, N363S: $n=2, \operatorname{Bcl1}: n=14$ ), while only one nonresponder patient had Bcl1 variant. None of these polymorphisms were found to be significantly associated to the therapeutic response rate.

Response to therapy was not associated with age and duration of thyroid or eye disease at any time point during therapy or follow-up. While no differences were observed at 6-8-weeks after therapy, the CAS improved more frequently in nonsmokers compared with smokers at 12-16 weeks $(P<0.045)$, but not at 24 weeks of follow-up. Twenty-four weeks after treatment, $11 / 58$ patients (19\%) had active GO, nine patients because of relapsing GO, and two who did not respond to therapy. Of the nine patients with GO reactivation, two received 16 IVGC infusions and six 12 IVGC infusions but the same cumulative dose (7.5 g). The number of patients with disease relapse among responders at $6-8$ weeks $(4 / 33,12.1 \%)$ did not differ from that among responders at 12-16 weeks of therapy $(5 / 33$, $15.1 \% ; P=\mathrm{NS})$. Patients not responding to therapy at 6-8 weeks had significantly higher basal levels of serum TRAb $(24.4 \pm 21.1 \mathrm{U} / \mathrm{l})$ when compared with responders $(15.4 \pm 20.4 \mathrm{U} / 1, P<0.01)$, but the changes in serum TRAb levels in response to treatment were not different in the

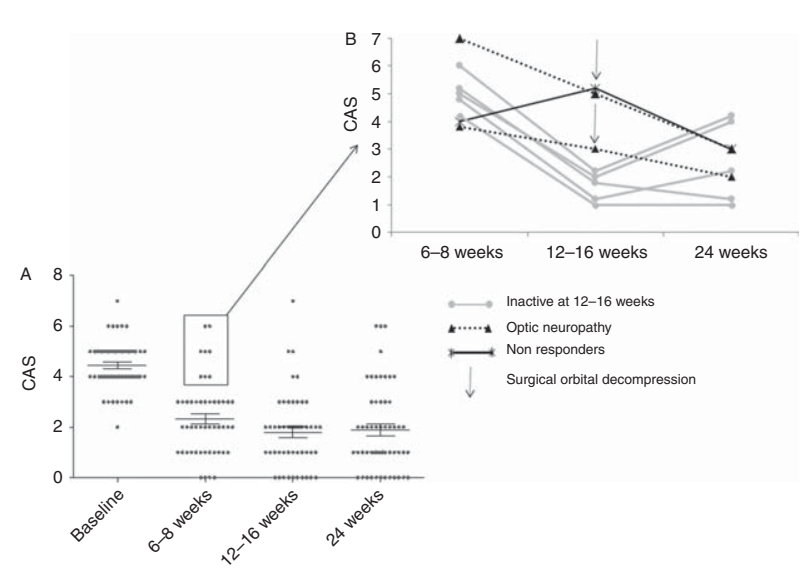

\section{Figure 2}

(A) Changes in the clinical activity score (CAS) in patient with active GO (CAS > 3/7) after IVGC. (B) Course of disease activity in eight patients not responding to IVGC at 6-8 weeks: five patients became inactive at 12-16 weeks, two developed DON and were surgically decompressed, and one did not respond. Two responders at 12-16 weeks relapsed at 24 weeks. A CAS $<4$ indicates disease inactivation. 
Table 2 Relationship between activity and severity of GO at the end of IVGC therapy (12-16 weeks).

\begin{tabular}{|c|c|c|c|c|}
\hline & \multicolumn{4}{|c|}{ Proptosis $^{a}$} \\
\hline & Improved & Unchanged & Worsened & Total \\
\hline \multicolumn{5}{|l|}{$\mathrm{CAS}^{\mathrm{b}}$} \\
\hline Improved & 10 & 26 & 3 & 39 \\
\hline Unchanged & 0 & 6 & 2 & 8 \\
\hline Worsened & 0 & 0 & 2 & 2 \\
\hline Total & 10 & 32 & 7 & 49 \\
\hline
\end{tabular}

two groups (not shown). Overall, we did not observe significant changes in proptosis, lid fissure, and eye motility after therapy. Interestingly, in about $25 \%$ of patients in whom therapy resulted in GO inactivation (CAS $<4 / 10$ ) at $6-8$ weeks, we observed a significant association of the CAS with the decrease in proptosis values in OD at $12-16$ weeks $(P<0.003)$ (Table 2$)$. Of the 17 patients showing constant diplopia at baseline, 11 improved and six did not change at 6-8 weeks after treatment (Fig. 3). At 24 weeks, no other patients showed improvement of diplopia, but one in fact worsened, probably due to fibrotic scarring of the eye muscles.

\section{Safety record}

Five patients $(8.6 \%)$ developed major side effects, and 23 patients (39.6\%) minor adverse events. Of patients with major reactions, two patients had a fourfold increase in serum liver enzymes, one developed diabetes, and two patients had major depression that required treatment. In none of patients did IVGC have to be withdrawn. Patients most frequently reported minor effects such as gastric disturbances and insomnia. Twenty-five of the 43 patients submitted to genetic analysis showed occurrence of side effects. GR polymorphisms were found in ten of these patients (10/25, 40\%) (E22R/E23K: $n=2$, N363S: $n=0$, Bcl1: $n=8)$. None of the GR polymorphisms described were found to be significantly associated with the occurrence of side effects.

\section{Discussion}

In this work, we have studied the time of response to IVGC therapy in patients with moderate-severe GO and whether the inter-individual variability of either therapeutic response or induced-toxicity could be associated with the expression of GR variants.
IVGC therapy has been shown to inactivate GO in about $70 \%$ of patients, a figure consistent with what was reported by Kahaly et al. (3) and, very recently, by a large randomized controlled multicenter study conducted by EUGOGO centers in Europe (4). The novel observation of our study is that about $70 \%$ of patients had inactive disease in response to IVGC as early as 6-8 weeks after the beginning of therapy and that only another $10 \%$ of patients attained GO inactivation with the continuation of therapy until 12-16 weeks. In addition, the rate of the therapeutic response and that of GO relapse did not appear to be significantly associated to either IVGC dose or duration of therapy.

The variability of IVGC therapeutic efficacy or induced-toxicity has been suggested by various groups to be associated with the expression of GR variants, which may be involved in the modulation of GC sensitivity and tolerability. The natural variability of the DNA sequence within the NR3C1 gene, localized on chromosome 5q31-q32, affects the activity of GC receptors (GR). The structural domain of the GR determines the biological functions of the functional domains. GR mutations determine either a modification of the secondary and tertiary domain structures of the receptor or an alteration of transcription initiation and stability of the mRNA. In diseases such as rheumatoid arthritis, leukemia, graft rejection, and many others, glucocorticoid resistance is

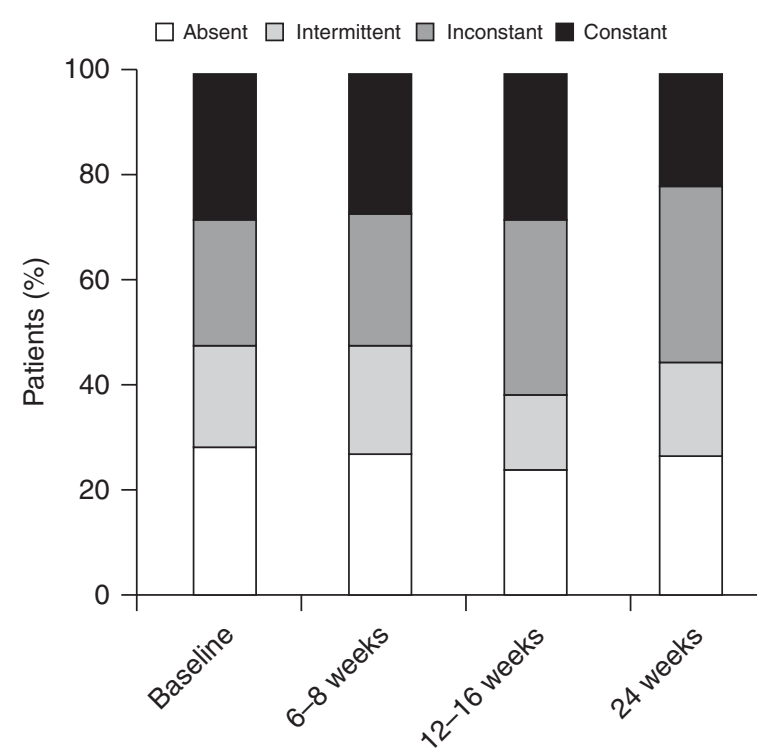

Figure 3

Change in the proportion of patients with different degrees of diplopia according to the Gorman score during IVGC therapy and follow-up $(P=\mathrm{NS})$. 
frequently observed; this situation has been reported in as many as $50 \%$ of patients who present with severe and difficult-to-treat asthma $(17,18)$. In this study, we have not been able to find GR variants that were significantly associated with either the therapeutic response or the occurrence of adverse events. It is though possible that our cohort of patients was too small to detect significance for a genetic association study, as the frequency of GR polymorphisms is known to be very low.

Among the factors influencing the therapeutic response, we observed that serum TRAb levels were higher at each timepoint in nonresponders compared with responders, in agreement with the data reported by Eckstein et al. (19) and decreased significantly in all patients, suggesting that IVGC also have an immunosuppressive effect on anti-thyroid antibody production. However, the slope of the decreasing curve of serum TRAb was not different in responders and nonresponders, suggesting that the efficacy of IVGC does depend on baseline TRAb levels but not on their effect on autoantibodies levels. Smoking has also been found to have a negative influence, as the majority of patients who improved at the end of therapy was not smokers, as previously suggested by Eckstein et al (20). This once more underscores the importance of smoking cessation as a primary therapeutic step.

Although overall parameters of GO severity were not significantly improved by treatment, we have observed an interesting relationship between the inactivation of the disease and the reduction of proptosis values at the end of treatment in a significant proportion of patients. The significant reduction of proptosis in about $25 \%$ of patients with inactive disease at the end of therapy and the lack of GO relapse in these patients suggest that the decrease in proptosis may be associated with an early and definitive response to IVGC therapy. The fact that this finding was significant only in one eye (OD) may simply reflect statistical clustering of a more frequent asymmetric presentation of GO (21), as a result of a different degree of immune infiltrates between the two orbits. Although in this study, IVGC is not associated with an overall improvement of eye motility, $\sim 65 \%$ of patients who had constant diplopia before therapy had improvement of eye motility as a response to IVGC as early as $6-8$ weeks after therapy, with no further improvement at 6 months. This finding is clinically relevant as these patients did not eventually need rehabilitative surgery.

IVGC therapy was well tolerated and none of the recorded side effects required IVGC discontinuation. Patients were carefully assessed before treatment, in order to identify even subclinical liver dysfunction and were never subjected to a cumulative dose of IVGC exceeding $8 \mathrm{~g}$ methylprednisolone, which has been reported to be reasonably safe (13).

The results of this study suggest that when deciding for therapy, it is important to be able to identify patients poorly responsive or unresponsive at all to IVGC, which may account for as many as $20-30 \%$ of active moderate-severe GO patients. The dose of methylprednisolone is an important parameter, as shown in a recent EUGOGO study (i.e., 4) and indeed, higher doses of methylprednisolone have been shown to be more effective compared with low doses and must be used for patients with active and more severe GO. On the other hand, this study shows that most patients with active GO respond to IVGC as early as 6-8 weeks of therapy, in particular those who are not smokers. Based on our findings, the optimal course of IVGC to be administered to patients with active GO may be decided by observing the therapeutic response at 6-8 weeks of high dose of methylprednisolone. At that point, if patients do not respond to treatment, combination therapy with another anti-inflammatory or immunosuppressive therapy such as orbital radiotherapy (2), cyclosporine (22), or mycophenolate mofetil (a study is currently ongoing within the EUGOGO centers) might be the option. The biological agent rituximab has also been shown to be effective by several groups $(23,24$, $25,26)$, and although promising, this therapy awaits confirmation by two ongoing randomized controlled studies.

In conclusion, although until now IVGC has been the therapy of choice for active moderate-severe GO, a more individualized treatment has to be sought in order to provide more effective treatment to as many as $20 \%$ of patients who may be only temporarily responsive or not responsive.

\section{Declaration of interest}

The authors declare that there is no conflict of interest that could be perceived as prejudicing the impartiality of the research reported.

\section{Funding}

This work is supported by Fondazione Cà Granda, IRCCS, Milano, Italy.

\section{References}

1 Kahaly GJ, Shimony O, Gellman YN, Lytton SD, Eshkar-Sebban L, Rosenblum N, Refaeli E, Kassem S, Ilany J \& Naor D. Regulatory T-cells in Graves' orbitopathy: baseline findings and immunomodulation by anti-T lymphocyte globulin. Journal of Clinical Endocrinology and Metabolism 201196 422-429. (doi:10.1210/jc.2010-1424)

2 Marcocci C, Bartalena L, Tanda ML, Manetti L, Dell'Unto E, Rocchi R, Barbesino G, Mazzi B, Bartolomei MP, Lepri P et al. Comparison of 
the effectiveness and tolerability of intravenous or oral glucocorticoids associated with orbital radiotherapy in the management of severe Graves' ophthalmopathy: results of a prospective, singleblind, randomized study. Journal of Clinical Endocrinology and Metabolism 2001 86 3562-3567. (doi:10.1210/jc.86.8.3562)

3 Kahaly GJ, Pitz S, Hommel G \& Dittmar M. Randomized, single-blind trial of intravenous versus oral steroid monotherapy in Graves' orbitopathy. Journal of Clinical Endocrinology and Metabolism 200590 5234-5240. (doi:10.1210/jc.2005-0148)

4 Bartalena L, Krassas GE, Wiersinga W, Marcocci C, Salvi M, Daumerie C, Bournaud C, Stahl M, Sassi L, Veronesi G et al. Efficacy and safety of three different cumulative doses of intravenous methylprednisolone for moderate to severe and active Graves' orbitopathy. Journal of Clinical Endocrinology and Metabolism 201297 4454-4463. (doi:10.1210/jc.2012-2389)

5 Hart RH, Kendall-Taylor P, Crombie A \& Perros P. Early response to intravenous glucocorticoids for severe thyroid-associated ophthalmopathy predicts treatment outcome. Journal of Ocular Pharmacology and Therapeutics 200521 328-336. (doi:10.1089/jop.2005.21.328)

6 Marinó M, Morabito E, Brunetto MR, Bartalena L, Pinchera A \& Marocci C. Acute and severe liver damage associated with intravenous glucocorticoid pulse therapy in patients with Graves' ophthalmopathy. Thyroid 200414 403-406. (doi:10.1089/105072504774193276)

7 Le Moli R, Baldeschi L, Saeed P, Regensburg N, Mourits MP \& Wiersinga WM. Determinants of liver damage associated with intravenous methylprednisolone pulse therapy in Graves' ophthalmopathy. Thyroid 200717 357-362. (doi:10.1089/thy.2006.0267)

8 Hiromatsu Y. Steroid therapy for Graves' ophthalmopathy. Nihon Rinsho $2006642279-2285$.

9 Wichary H \& Gasińska T. Methylprednisolone and hepatotoxicity in Graves' ophthalmopathy. Thyroid 201222 64-69. (doi:10.1089/thy. 2010.0158)

10 Gergics P, Patocs A, Majnik J, Balogh K, Szappanos A, Toth M \& Racz K. Detection of the Bcl I polymorphism of the glucocorticoid receptor gene by single-tube allele-specific polymerase chain reaction. Journal of Steroid Biochemistry and Molecular Biology 2006100 161-166. (doi:10.1016/j.jsbmb.2006.04.004)

11 Majnik J, Patocs A, Balogh K, Toth M \& Racz K. A rapid simple method for detection of Asn363Ser polymorphism of the human glucocorticoid receptor gene. Journal of Steroid Biochemistry and Molecular Biology 2004 92 465-468. (doi:10.1016/j.jsbmb.2004.08.005)

12 Karl M, Lamberts SWJ, Detera-Wadleigh SD, Encio IJ, Stratakis CA, Hurley DM, Accili D \& Chrousos GP. Familial glucocorticoid resistance caused by a splice site deletion in the human glucocorticoid receptor gene. Journal of Clinical Endocrinology and Metabolism 199376 683-689. (doi:10.1210/jc.76.3.683)

13 Eipel M, Németh K, Török D, Csordás K, Hegyi M, Ponyi A, Ferenczy A, Erdélyi DJ, Csóka M \& Kovács GT. The glucocorticoid receptor gene polymorphism N363S predisposes to more severe toxic side effects during pediatric acute lymphoblastic leukemia (ALL) therapy. International Journal of Hematology 201397 216-222. (doi:10.1007/ s12185-012-1236-1)

14 Bartalena L, Baldeschi L, Dickinson A, Eckstein A, Kendall-Taylor P, Marcocci C, Mourits M, Perros P, Boboridis K, Boschi A et al. Consensus statement of the European Group on Graves' orbitopathy (EUGOGO) on management of GO. European Journal of Endocrinology $2008 \mathbf{1 5 8}$ 273-285. (doi:10.1530/EJE-07-0666)

15 Dickinson AJ \& Perros P. Controversies in the clinical evaluation of active thyroid-associated orbitopathy: use of a detailed protocol with comparative photographs for objective assessment. Clinical Endocrinology 200155 283-303. (doi:10.1046/j.1365-2265.2001.01349.x)

16 Mourits MP, Prummel MF, Wiersinga WM \& Koorneef L. Clinical activity score as a guide in the management of patients with Graves' ophthalmopathy. Clinical Endocrinology 1997 47 9-14. (doi:10.1046/j. 1365-2265.1997.2331047.x)

17 Lane S \& Lee T. Mechanisms of corticosteroid resistance in asthmatic patients. International Archives of Allergy and Immunology 1997113 193-195. (doi:10.1159/000237544)

18 Panek M, Pietras T \& Kupryś-Lipińska I. The analysis of the factors influencing the development of glucocorticoid resistance in the etiopathogenesis of severe bronchial asthma. Postepy Biochemii 201056 373-382.

19 Eckstein AK, Plicht M, Hildegard L, Neuhauser M, Mann K, Lederbogen S, Heckmann C, Esser J \& Morgenthaler NG. TSH-receptor autoantibodies are independent risk farctors for Graves' ophthalmopathy and help to predict severity and outcome of the disease. Journal of Clinical Endocrinology and Metabolism 200691 3464-3470. (doi:10.1210/ jc.2005-2813)

20 Eckstein A, Quadbeck B, Mueller G, Rettenmeier AW, Hoermann R, Mann K, Steuhl P \& Esser J. Impact of smoking on the response to treatment of thyroid associated ophthalmopathy. British Journal of Ophthalmology 200387 773-776. (doi:10.1136/bjo.87.6.773)

21 Burch HB \& Wartofsky L. Graves' ophthalmopathy: current concepts regarding pathogenesis and management. Endocrine Reviews 199314 747-793. (doi:10.1210/edrv-14-6-747)

22 Prummel MF, Mourits MP, Berghout A, Krenning EP, van der Gaag R, Koornneef L \& Wiersinga WM. Prednisone and cyclosporine in the treatment of severe Graves' ophthalmopathy. New England Journal of Medicine 1989321 1353-1359. (doi:10.1056/NEJM198911163212002)

23 Salvi M, Vannucchi G, Campi I, Currò N, Dazzi D, Simonetta S, Bonara P, Rossi S, Sina C, Guastella C et al. Treatment of Graves' disease and associated ophthalmopathy with the anti-CD20 monoclonal antibody rituximab: an open study. European Journal of Endocrinology 2007156 33-40. (doi:10.1530/eje.1.02325)

24 El Fassi D, Nielsen CH, Bonnema SJ, Hasselbalch HC \& Hegedüs L. B lymphocyte depletion with the monoclonal antibody rituximab in Graves' disease: a controlled pilot study. Journal of Clinical Endocrinology and Metabolism 200792 1769-1772. (doi:10.1210/jc.2006-2388)

25 Khanna D, Chong KK, Afifiyan NF, Hwang CJ, Lee DK, Garneau HC, Goldberg RA, Darwin CH, Smith TJ \& Douglas RS. Rituximab treatment of patients with severe, corticosteroid-resistant thyroid-associated ophthalmopathy. Ophthalmology 2010117 133-139. (doi:10.1016/j. ophtha.2009.05.029)

26 Mitchell AL, Gan EH, Morris M, Johnson K, Neoh C, Dickinson AJ, Perros P \& Pearce SH. The effect of B cell depletion therapy on anti-TSH receptor antibodies and clinical outcome in glucocorticoid refractory Graves' orbitopathy. Clinical Endocrinology 201379 437-442. (doi:10.1111/cen.12141)
Received 26 July 2013

Revised version received 25 September 2013

Accepted 14 October 2013 